\title{
Improved Lesion Detection by Using Axial T2-Weighted MRI with Full Spinal Cord Coverage in Multiple Sclerosis
}

\author{
(D) S. Galler, (D).-P. Stellmann, (D).L. Young, (DD. Kutzner, (D). Heesen, DJ. Fiehler, and (D) Siemonsen
}

\begin{abstract}
BACKGROUND AND PURPOSE: Identification of lesions in specific locations gains importance in multiple sclerosis imaging diagnostic criteria. In clinical routine, axial scans are usually exclusively obtained to depict the cervical spinal cord or used to confirm suspected lesions on sagittal scans. We sought to evaluate the detection rate for MS lesions on axial T2WI scans with full spinal cord coverage in comparison with sagittal scans.
\end{abstract}

MATERIALS AND METHODS: One hundred fifteen patients with definite or suspected MS underwent an MR imaging examination including 3-mm sagittal and 3.5-mm axial T2-weighted images with full spinal cord coverage. T2WI lesions were identified on axial and sagittal scans independently by 2 raters. Axial diameter, craniocaudal extension, lesion intensity, and location were analyzed.

RESULTS: Four hundred forty-nine of 509 (88.2\%) lesions were detected on axial and 337/509 (66.2\%) on sagittal scans. Only $277 / 449$ $(61.7 \%)$ axial lesions were also detected on sagittal images. The number of lesions visible on sagittal and axial images was dependent on the axial lesion diameter $(P<.001)$.

CONCLUSIONS: Axial T2WI scans with full spinal cord coverage showed 22\% more lesions in patients with MS in comparison with sagittal scans, especially for lesions with small axial diameters. We suggest including biplanar spinal MR imaging with full spinal cord coverage for lesion detection in MS in clinical routine and for clinical studies.

ABBREVIATIONS: $A D=$ axial diameter; $C C E=$ craniocaudal extension

M ultiple sclerosis is a chronic inflammatory disease, considered the most common demyelinating process involving the central nervous system. ${ }^{1}$ The diagnosis requires typical clinical findings in addition to the evidence of lesions in the CNS disseminated in time and space seen on MR imaging of the brain or spinal cord. ${ }^{2}$ While the diagnostic focus of most multiple sclerosis studies is still based on MR imaging of the brain, several studies

Received July 29, 2015; accepted after revision November 3.

From the Departments of Diagnostic and Interventional Neuroradiology (S.G., D.K., J.F., S.S.) and Neurology (J.-P.S., K.L.Y., C.H.), and the Institute of Neuroimmunology and Multiple Sclerosis (J.-P.S., K.L.Y., C.H., S.S.), University Medical Center HamburgEppendorf, Hamburg, Germany.

This study was supported by the Federal Ministry of Education and Research (S.S., J.-P.S., C.H.; Proposal/Contract 0315610-0315620 NEU2).

Paper previously presented at: American Society of Neuroradiology Annual Meeting and the Foundation of the ASNR Symposium, April 25-30, 2015; Chicago,

Illinois.

Please address correspondence to Stephanie Galler, MD, Department of Diagnostic and Interventional Neuroradiology, University Medical Center Hamburg-Eppendorf, Martinistr 52, 20246 Hamburg, Germany; e-mail: s.galler@uke.de

- Indicates open access to non-subscribers at www.ajnr.org

三 Indicates article with supplemental on-line table.

http://dx.doi.org/10.3174/ajnr.A4638 have revealed spinal cord lesions in $75 \%-90 \%$ of patients with clinically diagnosed MS. ${ }^{3-6}$ As many as $20 \%$ of spinal MS lesions are isolated, without coexisting brain lesions. ${ }^{1}$

Spinal cord abnormalities seen on MR imaging were incorporated into the McDonald Diagnostic Criteria for MS in 2005. 7,8 Since the revision of the McDonald Diagnostic Criteria for MS in $2010,{ }^{9}$ they have gained even more importance because better spinal cord lesion detection potentially impacts the recognition of the dissemination of MS lesions in space.

In 2006, a consortium of MS centers published consensus guidelines with a standardized MR imaging protocol for spinal cord imaging in MS, recommending a 3-plane scout; a pre- and postcontrast-enhanced sagittal T1; a pre-contrast-enhanced sagittal FSE proton-density/T2; and additionally, only in case of suspected lesions, a pre-contrast-enhanced axial FSE proton-density/T2 and a post-contrast-enhanced axial T1 $1{ }^{10}$ In the clinical routine of MS diagnostics, axial scans are typically obtained exclusively with coverage of the cervical spinal cord or are used to confirm suspected lesions in sagittal scans. This is mainly due to the long scanning time of axial scans with full spinal cord coverage.

So far in most MS studies, spinal cord lesions were evaluated 
and marked on the sagittal plane, ${ }^{11,12}$ while some groups included axial scans covering only the cervical spine $e^{13-15}$ and very few studies analyzed the axial and sagittal planes of the entire spinal cord. ${ }^{3,16}$ Also, lesion location and size were described on sagittal scans only or on axial scans covering the cervical spinal cord exclusively. ${ }^{15}$

We hypothesized that axial T2WI scans with full spinal cord coverage would detect more T2WI lesions in comparison with sagittal scans. We sought to evaluate detection rates for T2WI lesions on axial and sagittal scans in relation to the distribution and extent of spinal cord lesions in patients with MS. To our knowledge, this is the first study focusing on the clinical application of axial 3.5-mm scans with full spinal cord coverage. We used a sequence with reasonable duration, feasible in clinical routine.

\section{MATERIALS AND METHODS \\ Patients}

One hundred fifteen patients (81 women, 16-82 years of age; mean, 38 years; 34 men, $16-71$ years of age; mean, 38 years) with diagnosed or suspected MS, and clinically suspected or known spinal cord lesions were consecutively included in our study between April 2013 and February 2014. Patients were referred for MR imaging by our associated MS day hospital. The prospective study was approved by the local ethics review committee and is in accordance with the Declaration of Helsinki. Patients or their guardians provided written informed consent. All patients underwent an MR imaging examination of the spinal cord, including axial scans, with full spinal cord coverage.

\section{MR Imaging Protocol}

All examinations were performed on a 3T MR imaging scanner (Magnetom Skyra; Siemens, Erlangen, Germany) with a neck and spine multiarray coil including, among other sequences, an axial T2WI turbo spin-echo sequence (TR, 6600 ms; TE, 94 ms; FOV, $200 \mathrm{~mm}$; section thickness, $3.5 \mathrm{~mm}$; 50 sections without a section gap; acquisition time, 2 minutes and 53 seconds) in 2 slabs with full spinal cord coverage (including the tip of the odontoid process and the conus medullaris) and a sagittal T2WI turbo spinecho sequence (TR, 3650 ms; TE, 95 ms; FOV, 300 mm; section thickness, $3 \mathrm{~mm}$; 17 sections without a section gap; acquisition time, 1 minute and 55 seconds). An intersection cross-talk was avoided by interleave acquisitions.

\section{Lesion Evaluation}

Lesion Identification. Lesions were defined as macular signal alterations with a minimum axial diameter of $2 \mathrm{~mm}$ that could be clearly isolated from their surroundings and not mistaken for artifacts. Axial and sagittal T2-weighted images were analyzed by 2 experienced neuroradiologists on a medical workstation on calibrated, high-resolution monitors. In a first reading, sagittal scans were presented to both raters separately in random order and MS lesions were identified and marked by each rater. In a second reading, corresponding axial scans were presented to both raters in the same manner. There was an interval of 2 weeks between the readings. For both readings, raters were blinded to the patient name and all other clinical and imaging parameters, especially to rating results and images of corresponding axial or sagittal scans.
Table 1: $\kappa$ values and numbers of detected lesions on sagittal and axial images

\begin{tabular}{lccc}
\hline & R1 & R2 & $\boldsymbol{\kappa}$ Value \\
\hline Axial & 463 & 458 & 0.831 \\
Sagittal & 324 & 301 & 0.804 \\
Axial and sagittal & 248 & 235 & 0.841 \\
Total & 391 & 401 & \\
\hline
\end{tabular}

Note:-R1 indicates rater 1; R2, rater 2 .

The readings were performed with the same intensity windowing to ascertain comparability between raters and scans. After completion of the blinded reading, lesions that were found only in axial or sagittal scans were reevaluated and documented to assess whether the lesion could be identified retrospectively in the corresponding axial or sagittal plane.

Lesion Characterization. Each lesion was characterized according to its intensity, location in the axial plane (midline or lateral), and position (cervical or thoracic) following the blinded reading. In addition, the craniocaudal extension (CCE) and axial diameter (AD) of each lesion were documented. To assess lesion extension in the axial plane, we measured the maximum lesion diameter, and lesions were stratified accordingly: AD1, 0.2-0.3 cm; AD2, $0.3-0.5 \mathrm{~cm}$; AD3, > $0.5 \mathrm{~cm}$.

Lesions were grouped according to their intensity $(\mathrm{I} 1=$ low intensity, I2 = intermediate intensity, $\mathrm{I} 3$ = high intensity relative to CSF).

\section{Statistical Analysis}

The interobserver agreement for T2 lesion detection in axial and sagittal scans was evaluated with the Cohen test, and magnitude guidelines were applied according to Landis and Koch. ${ }^{17}$ For further analysis, only lesions identified by both raters were included. Ordinal data were evaluated by using the $\chi^{2}$ test. All statistical analyses were conducted by using $\mathrm{R}$ statistical computing software, Version 3.0.0 (http://www.r-project.org/).

\section{RESULTS}

\section{Interrater Agreement}

Ratings for detection of lesions on sagittal and axial scans for raters 1 and 2 are shown in Table 1. Both raters detected more lesions on axial than on sagittal scans. The Cohen $\kappa$ value for interrater reliability was almost perfect $(\kappa=0.841)$ for lesion detection in axial and sagittal scans and only slightly lower $(\kappa=$ 0.804) for sagittal scans (Table 1).

\section{Lesion Evaluation}

In total, 509 lesions were detected on axial or sagittal scans by both raters. Four hundred forty-four T2WI hyperintense spinal cord lesions were identified on axial scans, and 284 lesions, on sagittal scans during the first reading (Table 2). When scans were evaluated retrospectively, the number of lesions that were missed in the first but identified in the second reading was considerably higher on sagittal in comparison with axial scans (Fig 1). Overall, 449/509 (88.2\%) lesions were detected on axial scans, while $337 / 509$ (66.2\%) lesions were detected on sagittal scans. Of all lesions detected on axial scans, only 277/449 $(61.7 \%)$ were also detected on sagittal scans, while 60/337 $(17.8 \%)$ were detected only on sagittal scans without any cor- 
relation on axial scans, even in retrospective evaluation (Fig 1 and Table 2). Sample images are shown in Figs 2 and 3. The median number of lesions identified per patient was 2 (minimum, 0; maximum, 22 lesions). In 31/115 (27\%) patients, no spinal cord lesion was identified by both raters. In the $84 / 115$ (73\%) patients with at least 1 detected spinal cord T2 lesion, the median number of lesions was 6 , with a minimum of 1 lesion per patient and a maximum number of 22 .

\section{Lesion Location, Size, and Intensity}

There was no significant difference in the number of lesions located in the cervical or thoracic spinal cord $(P>.2)$ on axial or sagittal scans. The number of lesions detected only on sagittal but not on axial scans was significantly higher within the thoracic spinal cord than in the cervical cord $(P<.001)$ (Fig 4$)$. On axial scans, $185 / 449(41.2 \%)$ lesions were located in the

Table 2: Lesions in the cervical and thoracic spinal cord $^{\mathrm{a}}$

\begin{tabular}{lccc}
\hline & Total & $\begin{array}{c}\text { Total Including } \\
\text { Retrospective } \\
\text { Analysis }\end{array}$ & $\begin{array}{c}\text { Spinal Cord } \\
\text { Location: } \\
\text { Cervical/Thoracic }\end{array}$ \\
\hline Axial & 444 & 449 & $237 / 212$ \\
Sagittal & 284 & 337 & $158 / 179$ \\
Axial and sagittal & 219 & 277 & $141 / 136$ \\
Axial, not sagittal & 225 & 172 & $96 / 76$ \\
Sagittal, not axial & 65 & 60 & $17 / 43$ \\
Total & 509 & 509 & $254 / 255$ \\
\hline
\end{tabular}

a Data are the number of detected lesions in first and second readings by both raters. midline of the spinal cord, $128 / 449$ (28.5\%) on the left side, and $136 / 449(30.3 \%)$ on the right side. The rate of axial lesions also recognized on sagittal scans was significantly higher for lesions with an in-plane location in the midline $(126 / 185$, $68.1 \%)$ than for lesions with a lateral location $(151 / 264,57.2 \%$, $P>.001)$.

In addition, lesion identification did not seem to be dependent on the degree of lesion intensity. Lesions with high intensity on axial scans were also identified on sagittal scans in most cases, but the percentage of lesions with low intensity (I1) identified on axial and sagittal scans was only slightly lower in comparison with those with high intensity (I3) and slightly higher than the percentage of lesions with medium intensity (I2) (On-line Table).

The percentage of lesions detected on sagittal and axial scans seemed to be dependent on the axial lesion diameter. The larger the lesions, the higher were the percentages of axial lesions also detected on sagittal scans. Forty of 43 (93.0\%) of the lesions with an axial diameter $>0.5 \mathrm{~cm}$ (AD3) were also identified on sagittal scans, while only 49/106 (46.2\%) lesions in group $\mathrm{AD} 1$ were also detected on sagittal scans (On-line Table).

When we compared the CCE of lesions, lesions that were detected on axial and sagittal scans showed significantly larger CCEs $(P<.001)$ than those lesions that were only identifiable on axial scans (Fig 5). Thirty-eight of $60(63.3 \%)$ lesions only identified on sagittal scans presented with a CCE $\leqq 0.3 \mathrm{~cm}$.

\section{Lesion detection in retrospective analysis}

500

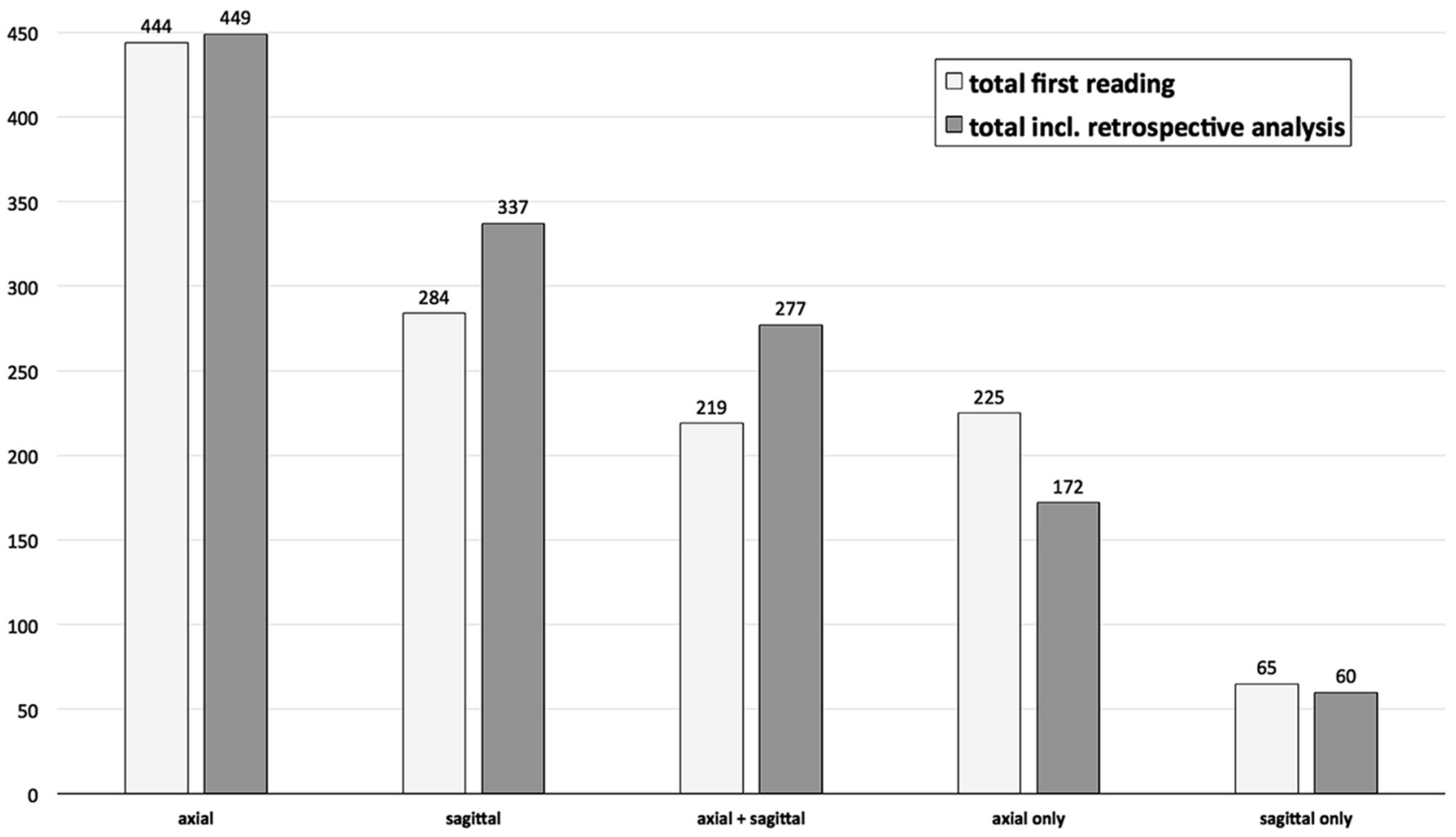

FIG 1. Graph displaying the total number of lesions detected on axial scans (axial), the total number of lesions detected on sagittal scans (sagittal), the number of lesions identified on both sagittal and axial scans (axial + sagittal), and the lesion number marked on axial (axial only) or sagittal (sagittal only) scans only without corresponding correlates in the other plane. Light gray bars indicate the results of the first reading, and dark gray bars correspond to lesion numbers, including the lesions that could only be identified when retrospectively analyzing the images. Incl indicates including. 

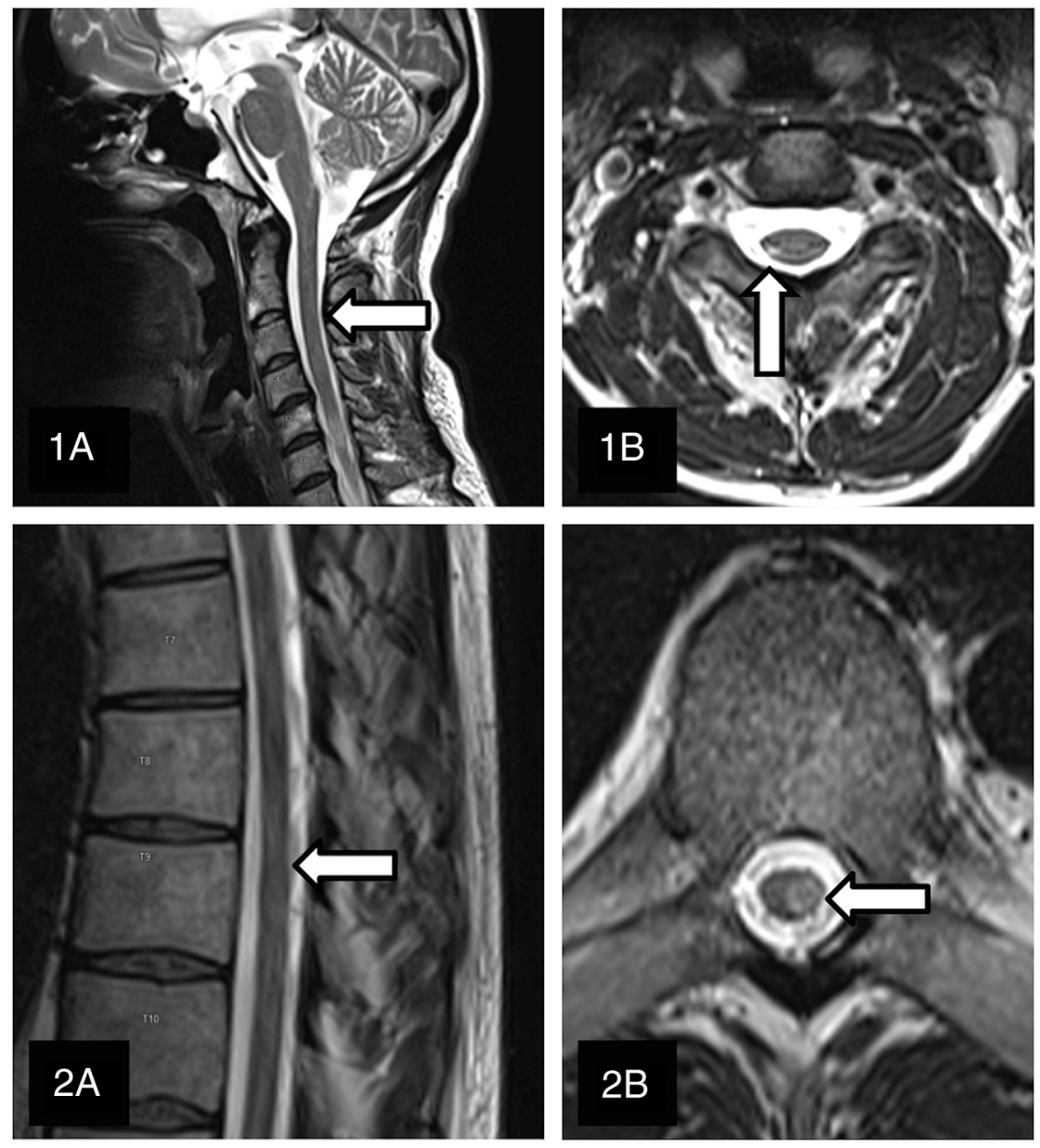

FIG 2. Examples of a cervical ( $7 A$ and $7 B$ ) and a thoracic ( $2 A$ and $2 B$ ) spinal cord lesion detected on axial scans but missed by both raters on corresponding sagittal scans.

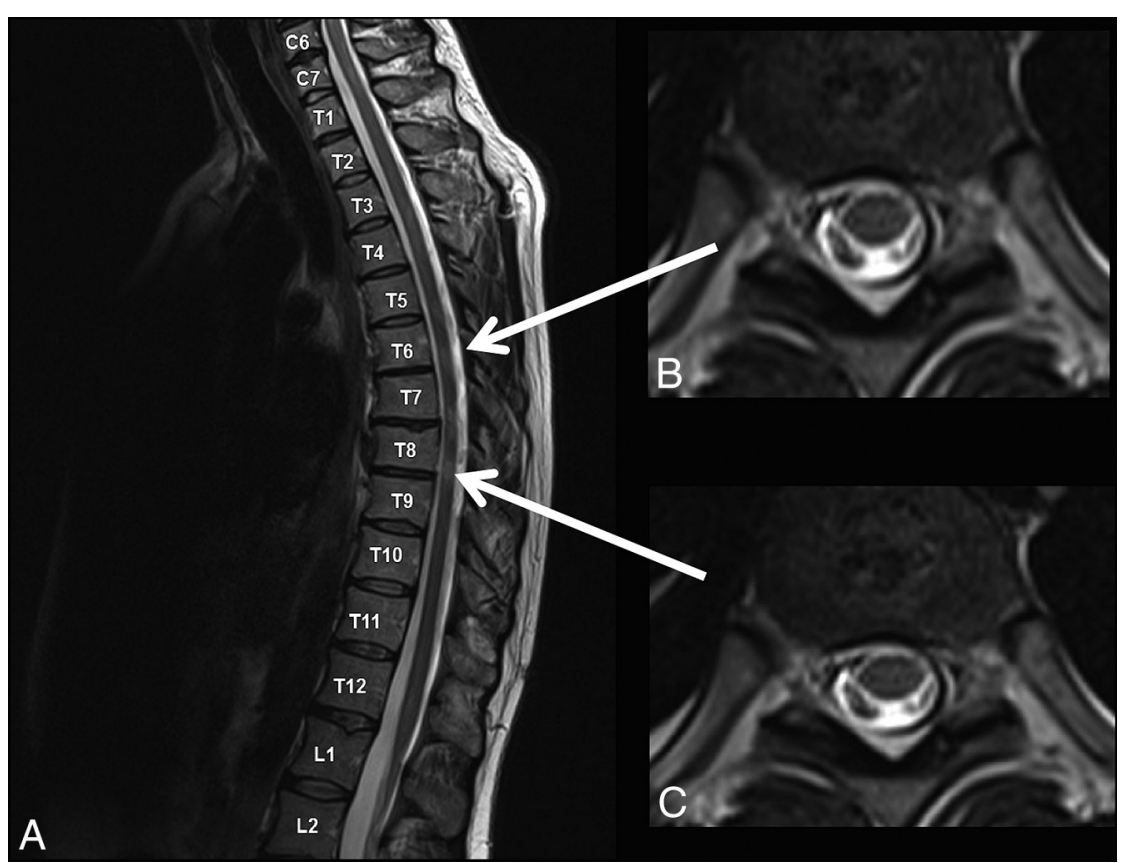

FIG 3. Examples of spinal cord lesions detected only on sagittal scans $(A)$ while missed on corresponding axial scans ( $B$ and $C)$.

\section{DISCUSSION}

Commonly, the spinal cord is assessed on sagittal scans with additional axial sections used to ascertain focal pathologies. It seems that this scanning protocol became clinical routine because a sagittal scan could be obtained much faster than a thorough coverage of the whole spinal cord with axial sections. In the present day, parallel imaging techniques with multiarray coils provide a timesaving scanning technique with axial sections with less patient movement and artifacts. ${ }^{18}$

The present investigation is the first MR imaging study using axial T2WI sequences with full spinal cord coverage in a cohort of patients with possible inflammatory spinal cord pathology from an MS clinic, to our knowledge. We compared the lesions found in sagittal and axial scans in number, location, signal intensity, and size by using the $\mathrm{AD}$ and the CCE. The overall acquisition time for a combined imaging study consisting of 2 slabs of axial T2WI scans (2:53 minutes) and sagittal T2WI scans (1:55 minutes) seems reasonable even for a routine clinical setting.

In comparison with the total number of lesions detected on sagittal scans, 172/ 337 (51\%) more lesions could only be detected on axial scans. Therefore, we focused on the analysis of all lesions depending on their size, location, and intensity, while previous studies mainly focused on the detection or exclusion of any spinal cord lesion identified on axial and sagittal scans., ${ }^{3,6}$

Previous studies gave the impression that focal spinal cord lesions in patients with MS are preferentially located in the cervical spinal cord. ${ }^{19-22}$ In 2004, Bot et $\mathrm{al}^{4}$ published a study with 353 spinal cord lesions in patients with MS; 56.4\% of these were located in the cervical spinal cord. In line with these previous studies, $52.7 \%$ of all spinal cord lesions detected in our study were located in the cervical segment. Notably, the percentage of lesions identified in the cervical spinal cord only slightly exceeded the percentage of thoracic lesions (47.3\%). Nevertheless, in both segments, cervical and thoracic, significantly more lesions were detected in the axial than on the sagittal planes. 


\section{Lesion detection and location}

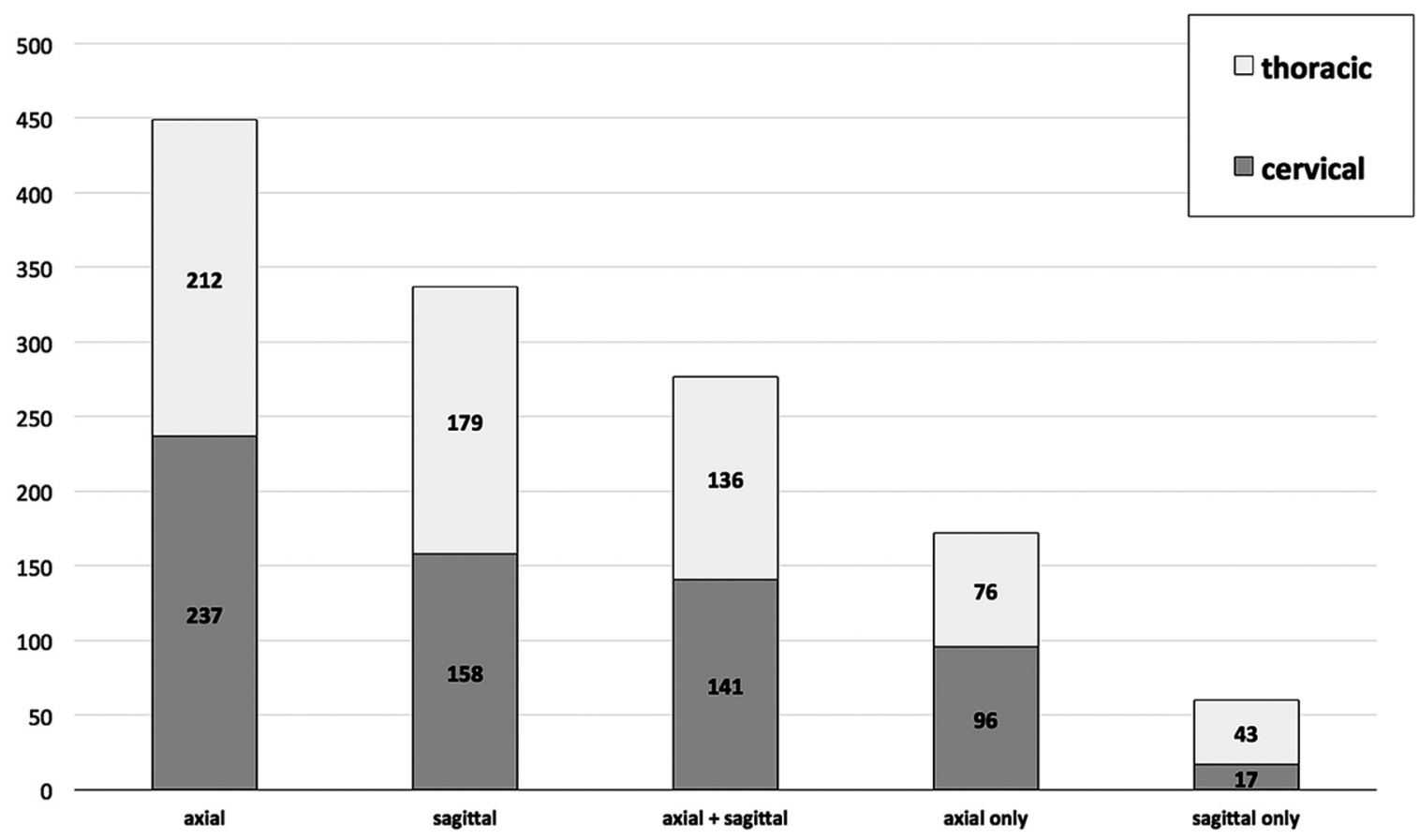

FIG 4. There was no significant difference in the number of lesions located in the cervical or thoracic spinal cord $(P>.2)$ on axial or sagittal scans. The number of lesions detected only on sagittal but not on axial scans was significantly higher in the thoracic spinal cord than in the cervical cord $(P<.001)$.

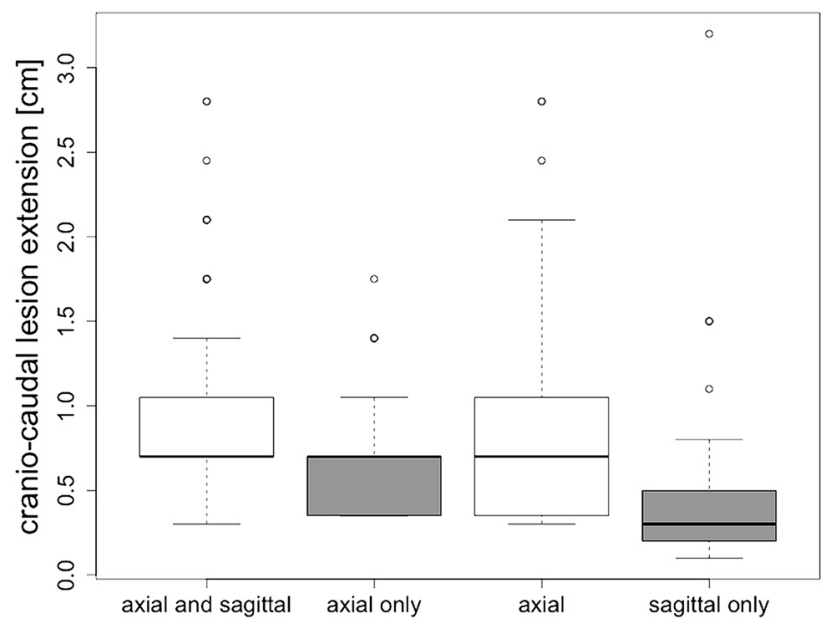

FIG 5. Boxplot comparing craniocaudal extension of lesions. Those lesions detected in axial and sagittal scans had a significantly larger extent $(P<.001)$ than those lesions that were only identifiable on axial scans (axial only).

Our findings demonstrate that the standardized MR imaging protocol for spinal cord imaging in MS, which recommends axial scans of suspicious lesions on sagittal planes only, ${ }^{10}$ leaves a high percentage of lesions undetected. In addition, the common clinical procedure to cover the cervical spine with an additional axial sequence seems to disregard, specifically, thoracic lesions.

Given that spinal cord lesions have very high specificity and sensitivity to differentiate MS and other neurologic diseases, missing every third lesion in clinical practice is not acceptable. In ad- dition, MR imaging is the only established biomarker to monitor disease activity and treatment response in MS. ${ }^{23}$ Two or more new T2 lesions on MR imaging of the brain are considered predictive of relapses and disability progression (ie, poor treatment response to immunotherapy). ${ }^{24}$ Currently, several treatments for MS are available. Their effectiveness is measured by their ability to decrease disease activity assessed with clinical scores and MR imaging. Therefore, detecting new lesions, also in the spinal cord, is crucial for treatment decisions. ${ }^{25}$

In line with authors of previous MR imaging and pathologic studies, we found lesions only infrequently in the anterior columns of the spinal cord. ${ }^{26-28}$ In addition, most of the detected lesions on sagittal and axial planes were located in the lateral columns of the spinal cord. Lateral cord lesions were likewise detected on sagittal scans in only $57.2 \%$ of the cases. Reasons for missing lesions on sagittal scans might be the section thickness of $3 \mathrm{~mm}$ and partial volume effects.

Accordingly, especially, lesions with a small in-plane extension (AD) could not be identified in sagittal planes. In addition, sagittal lesions that were missed on axial scans had significantly lower CCE. This might be explained by the axial section thickness of 3.5 $\mathrm{mm}$ that was used in this study, which is small compared with previous trials reporting axial section thicknesses of $6^{3}$ and 5 $\mathrm{mm}^{16}$

To our knowledge, measurements of the AD of single lesions have not been reported. Previous studies mainly focused on the CCE in sagittal scans. ${ }^{6}$

The lesion signal intensity did not seem to affect the likelihood of lesion identification. However, in some cases, lesions were 
more clearly identified on axial scans and only depicted as diffuse hyperintensities on the sagittal spinal cord images. This phenomenon has already been described in detail on postmortem MR imaging and histopathologic analyses. ${ }^{29}$ Without knowledge of corresponding axial scans, these lesions have been mainly considered artifacts.

A major limitation of this study in line with previous studies that compared MR imaging sequences for lesion detection ${ }^{30}$ was the lack of a reference standard for lesion presence. In the absence of postmortem pathologic data, which serve as the only reliable reference, we used consensus findings of 2 experienced raters in axial T2-weighted TSE images with full spinal cord coverage as a radiologic reference standard and compared these results with lesion detection on sagittal planes. In general, lesions were more clearly detectable and defined on axial than on sagittal T2WI scans. Therefore, we regarded axial scans as more reliable for lesion detection than corresponding sagittal scans. However, lesions identified only on sagittal scans might be missed because of their CCEs lying below or being equal to the section thickness of the axial sections.

The current diagnostic value of sagittal spinal MR images is limited mainly by partial volume effects, CSF pulsation artifacts, and section thickness of $3 \mathrm{~mm}$, resulting in a high number of lesions missed by the reader. Nevertheless, the sagittal scan should not be abandoned because lesions with small CCEs can also be missed on axial scans with 3-mm section thicknesses. Furthermore, when we retrospectively evaluated lesions that were only seen on axial scans in the first reading, $26.5 \%$ more lesions could be identified on both scans after the second reading and might therefore be regarded as reproducible.

\section{CONCLUSIONS}

In patients with MS, axial scans with full spinal cord coverage displayed considerably more T2WI lesions in comparison with sagittal scans in the cervical and in the thoracic spinal cord. This finding applies especially to lesions with a small axial diameter and lesions located in the lateral spinal cord. However, even large lesions can be overlooked when only assessed in the sagittal plane. Furthermore, axial scans with full spinal cord coverage allow quantification and volumetric analysis of spinal lesion load in patients with MS and might provide a new impulse for future MS studies.

We suggest biplanar spinal MR imaging with full axial spinal cord coverage as a comprehensive examination for lesion detection in MS in clinical routine and for clinical studies. The clinical implication of these additional findings needs to be confirmed and validated in future studies.

Disclosures: Stephanie Galler-RELATED: Grant: Federal Ministry of Education and Research, ${ }^{\star}$ Comments: S.S., J.-P.S., C.H., Proposal/Contract 0315610-0315620 NEU2. Jan-Patrick Stellmann—UNRELATED: Grants/Grants Pending: Biogen, ${ }^{\star}$ Merck Serono*; Payment for Lectures (including service on Speakers Bureaus): Biogen*; Travel/Accommodations/Meeting Expenses Unrelated to Activities Listed: Biogen, Novartis. Kim Lea Young - RELATED: Grant: Federal Ministry of Education and Research.* Daniel Kutzner-RELATED: Grant: Federal Ministry of Education and Research.* Christoph Heesen—RELATED: Grant: German Ministry of Education and Research, ${ }^{*}$ Comments: Grant to develop MRI platform for MS as an outcome for treatment studies. Jens Fiehler-UNRELATED: Payment for Lectures (including service on Speakers Bureaus): Siemens. *Money paid to the institution.

\section{REFERENCES}

1. Noseworthy JH, Lucchinetti C, Rodriguez M, et al. Multiple sclerosis. N Engl J Med 2000;343:938-52 CrossRef Medline

2. Inglese M. Multiple sclerosis: new insights and trends. AJNR Am J Neuroradiol 2006;27:954-57 Medline

3. Weier K, Mazraeh J, Naegelin Y, et al. Biplanar MRI for the assessment of the spinal cord in multiple sclerosis. Mult Scler 2012;18: 1560-69 CrossRef Medline

4. Bot JC, Barkhof F, Polman CH, et al. Spinal cord abnormalities in recently diagnosed MS patients: added value of spinal MRI examination. Neurology 2004;62:226-33 CrossRef Medline

5. Nijeholt GJ, van Walderveen MA, Castelijns JA, et al. Brain and spinal cord abnormalities in multiple sclerosis: correlation between MRI parameters, clinical subtypes and symptoms. Brain 1998; 121(pt 4):687-97 CrossRef Medline

6. Qiu W, Raven S, James I, et al. Spinal cord involvement in multiple sclerosis: a correlative MRI and high-resolution HLA-DRB1 genotyping study. J Neurol Sci 2011;300:114-19 CrossRef Medline

7. Korteweg T, Barkhof F, Uitdehaag BM, et al. How to use spinal cord magnetic resonance imaging in the McDonald diagnostic criteria for multiple sclerosis. Ann Neurol 2005;57:606-07 CrossRef Medline

8. Polman CH, Reingold SC, Edan G, et al. Diagnostic criteria for multiple sclerosis: 2005 revisions to the "McDonald Criteria." Ann Neurol 2005;58:840-46 CrossRef Medline

9. Polman CH, Reingold SC, Banwell B, et al. Diagnostic criteria for multiple sclerosis: 2010 revisions to the McDonald criteria. Ann Neurol 2011;69:292-302 CrossRef Medline

10. Simon JH, Li D, Traboulsee A, et al. Standardized MR imaging protocol for multiple sclerosis: Consortium of MS Centers consensus guidelines. AJNR Am J Neuroradiol 2006;27:455-61 Medline

11. Lukas C, Sombekke MH, Bellenberg B, et al. Relevance of spinal cord abnormalities to clinical disability in multiple sclerosis: MR imaging findings in a large cohort of patients. Radiology 2013;269:542-52 CrossRef Medline

12. Bot JC, Blezer EL, Kamphorst W, et al. The spinal cord in multiple sclerosis: relationship of high-spatial-resolution quantitative MR imaging findings to histopathologic results. Radiology 2004;233: 531-40 CrossRef Medline

13. Riederer I, Karampinos DC, Settles M, et al. Double inversion recovery sequence of the cervical spinal cord in multiple sclerosis and related inflammatory diseases. AJNR Am J Neuroradiol 2015;36: 219-25 CrossRef Medline

14. Raz E, Bester M, Sigmund EE, et al. A better characterization of spinal cord damage in multiple sclerosis: a diffusional kurtosis imaging study. AJNR Am J Neuroradiol 2013;34:1846-52 CrossRef Medline

15. Kearney H, Altmann DR, Samson RS, et al. Cervical cord lesion load is associated with disability independently from atrophy in MS. Neurology 2015;84:367-73 CrossRef Medline

16. Nair G, Absinta M, Reich DS. Optimized T1-MPRAGE sequence for better visualization of spinal cord multiple sclerosis lesions at $3 \mathrm{~T}$. AJNR Am J Neuroradiol 2013;34:2215-22 CrossRef Medline

17. Landis JR, Koch GG. The measurement of observer agreement for categorical data. Biometrics 1977;33:159-74 CrossRef Medline

18. Pruessmann KP, Weiger M, Scheidegger MB, et al. SENSE: sensitivity encoding for fast MRI. Magn Reson Med 1999;42:952-62 Medline

19. Kidd D, Thorpe JW, Thompson AJ, et al. Spinal cord MRI using multi-array coils and fast spin echo, II: findings in multiple sclerosis. Neurology 1993;43:2632-37 CrossRef Medline

20. Bot JC, Barkhof F, Lycklama à Nijeholt G, et al. Differentiation of multiple sclerosis from other inflammatory disorders and cerebrovascular disease: value of spinal MR imaging. Radiology 2002;223: 46-56 CrossRef Medline

21. Honig LS, Sheremata WA. Magnetic resonance imaging of spinal cord lesions in multiple sclerosis. J Neurol Neurosurg Psychiatry 1989;52:459-66 CrossRef Medline 
22. Tartaglino LM, Friedman DP, Flanders AE, et al. Multiple sclerosis in the spinal cord: MR appearance and correlation with clinical parameters. Radiology 1995;195:725-32 CrossRef Medline

23. Dobson R, Rudick RA, Turner B, et al. Assessing treatment response to interferon- $\boldsymbol{\beta}$ : is there a role for MRI? Neurology 2014;82:248-54 CrossRef Medline

24. Hauser SL, Chan JR, Oksenberg JR. Multiple sclerosis: prospects and promise. Ann Neurol 2013;74:317-27 CrossRef Medline

25. Rotstein DL, Healy BC, Malik MT, et al. Evaluation of no evidence of disease activity in a 7-year longitudinal multiple sclerosis cohort. JAMA Neurol 2015;72:152-58 CrossRef Medline

26. Gilmore CP, Geurts JJ, Evangelou N, et al. Spinal cord grey matter lesions in multiple sclerosis detected by post-mortem high field MR imaging. Mult Scler 2009;15:180-88 CrossRef Medline
27. Ikuta F, Zimmerman HM. Distribution of plaques in seventy autopsy cases of multiple sclerosis in the United States. Neurology 1976;26(6 pt 2):26-28 Medline

28. Adams RD, Kubik CS. The morbid anatomy of the demyelinative disease. Am J Med 1952;12:510 - 46 CrossRef Medline

29. Bergers E, Bot JC, van der Valk P, et al. Diffuse signal abnormalities in the spinal cord in multiple sclerosis: direct postmortem in situ magnetic resonance imaging correlated with in vitro high-resolution magnetic resonance imaging and histopathology. Ann Neurol 2002;51:652-56 CrossRef Medline

30. Martin N, Malfair D, Zhao Y, et al. Comparison of MERGE and axial T2-weighted fast spin-echo sequences for detection of multiple sclerosis lesions in the cervical spinal cord. AJR Am J Roentgenol 2012;199:157-62 CrossRef Medline 